\title{
Preface: Special Session SpS14 Communicating Astronomy with the Public for Scientists
}

Astronomers "have a responsibility to communicate their results and efforts with the public for the benefit of all", especially since most are publically funded. The importance of science communication has never been greater nor have the opportunities for communication of science to the public been larger. While astronomers are highly trained in carrying out scientific research, in general they are poorly equipped to communicate the excitement and results of their research to a broader audience. Generally, they do not have information about the various opportunities for communicating astronomy nor on the resources to help them and support them in communicating astronomy.

The primary goal of this Special Session 14 was to provide the interested astronomers with helpful hints and tips into effective communication with the public. The IAU General Assembly provided an ideal environment to attract astronomers interested in the public communication of astronomy.

Journalists and scientists often operate at opposite ends of the communication spectrum. Often scientists are disappointed about media coverage of their research, and journalists report frustration with the difficulties of describing and understanding important scientific findings. There is plenty of room for mistrust to build and problematic issues to arise. Some scientists are uncomfortable about participating in science communication but there are many good reasons why scientists should participate in public science communication:

- To expose the work of his/her specific community.

- To highlight a specific result.

- To highlight the work of an institution.

- To highlight the work of a group.

- To highlight individual efforts (which is perfectly all right!).

- To acknowledge a sponsor.

- To do a favour to the scientific community as a whole (a sense of duty).

SpS 14 featured key speakers knowledgeable in various areas of science communication. These invited speakers shared their best practices with the attendees. SpS 14 also featured many contributed talks from an extremely wide geographic area. These talks exposed the audience to the multitide of communication opportunities that are available to anyone interested in communicating astronomy with the public.

A very powerful example of astronomy communication was presented early on the first day. Members of the Tenpla project, visited Iwate prefecture, one of the areas most severely damaged by the Great Tohoku earthquake. They held star parties, workshops, plays and talks at several evacuation centers. Even in such difficult circumstances, people responded very positively to their efforts.

The same group also provided samples of astronomical toilet, in Japanese of course!, which provided much comic relief.

Dennis Crabtree and Lars Lindberg Christensen, co-chairs SOC 